\title{
Features of control of characteristics of radio engineering devices in the multi-wave mode of operation
}

\author{
Vladimir N. Pichugin* \\ Chuvash state University named I.N. Ulyanov, Cheboksary, Russia
}

\begin{abstract}
In multi-wave elements of radio devices at frequencies, $1,2, \ldots$, 10 times the operating frequency, conditions arise for the propagation of several types of waves. This leads to the dependence of the characteristics of electronic devices and, consequently, the parameters of frequency selectivity on the spatial structure (spatial spectrum, mode composition) of the electromagnetic signal at the input of the element under study. The presence of such" spatial selectivity" requires taking into account the features of the multi-wave mode in solving various problems of electromagnetic compatibility. The article is devoted to the actual problemthe development of new methods of experimental research and control of the characteristics of electromagnetic waves of radio devices operating in multi-wave mode. The solution of the problem of studying the impact of a plane electromagnetic wave on the emitters of the radio-thnical system is carried out. The analytical calculation of the electrodynamics effects in the multi-wave mode is carried out.
\end{abstract}

\section{Introduction}

In multi-wave elements of radio devices at frequencies, $1,2, \ldots, 10$ times higher than the operating frequency, conditions arise for the propagation of several types of waves. This leads to the dependence of the characteristics of electronic devices and, consequently, the parameters of frequency selectivity on the spatial structure (spatial spectrum, mode composition) of the electromagnetic signal at the input of the element under study. The presence of such "spatial selectivity" requires taking into account the features of the multiwave regime in solving various problems of electromagnetic compatibility $[1,2,3]$.

For certainty, under the radio engineering we will understand the linear portion of the feeder-th tract, having input and output shoulders in the form of segments of regular waveguides. We will assume the length of these segments sufficient for attenuation of nonpropagating types of waves and therefore limit ourselves to considering only propagating waves $[4,5]$.

\footnotetext{
${ }^{*}$ Corresponding author: vladimir_iii@mail.ru
} 


\section{The influence of the multiwave regime on the parameters of radio devices}

Under the accepted restrictions, the magnetic and electric fields of electromagnetic waves propagating in the arms in the direction to the node (incident waves) (- shoulder number) or in the opposite direction (reflected waves) on the cross section of the arm (reference plane) can be represented as a finite sum of the distributions of electric and magnetic fields of natural shoulder waves:

$$
\begin{gathered}
\left.E_{a i}(x, y)=\sum_{1}^{N_{i}} a_{i k} e_{a i k}(x, y), H_{a i}(x, y)=\sum_{1}^{N_{i}} a_{i k} h_{a i k}(x, y)\right), \\
E_{b i}(x, y)=\sum_{1}^{N_{i}} b_{i k} e_{b i k}(x, y), H_{b i}(x, y)=\sum_{1}^{N_{i}} b_{i k} h_{b i k}(x, y),
\end{gathered}
$$

where $e_{a i k}(x, y), e_{b i k}(x, y), h_{a i k}(x, y), h_{b i k}(x, y)$ - the distribution of electric and magnetic fields of their own type of waves propagating respectively in the direction of the antenna and Vice versa, in the direction of the plane of the shoulder; $N_{i}$ - the number of the propagating waves in the shoulder; $a_{u л}, b_{\text {uл }}$ - he complex expansion coefficients; $x, \mathrm{y}$ - the coordinates in the reference plane.

The specific type of natural shoulder waves is determined by the shape and size of the antenna cross section. The numbering of wave types can be arbitrary.

Thus, for the selected form of eigenwaves, the electromagnetic fields of incident and reflected waves from the antenna are fully characterized by a set of complex values $a_{u л}, b_{\text {uл }}$, which in the future will be called the modal composition of the input or output signal. Set of complex values

$$
a_{i k}^{u}=a_{i k} / \sqrt{\sqrt{\sum_{1}^{N_{i}}\left|a_{i k}\right|^{2}}} \quad b_{i k}^{u}=b_{i k} / \sqrt{\sqrt{\sum_{1}^{N_{i}}\left|b_{i k}\right|^{2}}}
$$

we shall call normalized medovym composition.

Due to the linearity of the antenna between $a_{j k}$ and $b_{j k}$ there is a linear relationship:

$$
b_{i k}=\sum_{i=1}^{2} \sum_{l=1}^{N_{i}} s_{k l}^{j i} a_{i k}, k=1,2 \ldots . . N_{j}, j=1,2, \ldots .
$$

where $s_{k l}^{j i}$-complex values characterizing the properties of this antenna system and being elements of the scattering matrix:

$$
S=\left(\begin{array}{cccc}
s_{11}^{11} & s_{1 N_{1}}^{11} & s_{11}^{21} & s_{1 N_{2}}^{12} \\
s_{N_{1} 1}^{11} & s_{N_{1} N_{1}}^{11} & s_{N_{1} 1}^{12} & s_{N_{1} N_{2}}^{12} \\
s_{11}^{21} & s_{1 N_{1}}^{21} & s_{11}^{22} & s_{1 N_{2}}^{22} \\
s_{N_{2} 1}^{21} & s_{N_{2} N_{1}}^{21} & s_{N_{2} 1}^{22} & s_{N_{2} N_{1}}^{22}
\end{array}\right) .
$$

Subject to (3) relation (2) you can write in the following compressed form:

$$
B=S \times A,
$$

where $A=\left(a_{11}, a_{12}, \ldots, a_{1 N_{1}}, a_{21}, \ldots, a_{2 N_{2}}\right)^{T}, B=\left(b_{11}, b_{12}, \ldots, b_{1 N_{1}}, b_{21}, \ldots b_{2 N_{2}}\right)^{T}$ - matrix-columns $(T$ transpose sign), or in a more convenient form for further presentation: $B=S_{11} A_{1}+S_{12} A_{2}, B_{2}=S_{21} A_{1}+S_{22} A_{2}$ - the blocks of the scattering matrix: 


$$
\begin{gathered}
S_{i j}=\left(\begin{array}{cc}
s_{11}^{i j} & s_{1 N_{j}}^{i j} \\
s_{N_{i 1}}^{i j} & s_{N_{i} N_{j}}^{i j}
\end{array}\right), \\
A_{i}=\left(a_{i 1}, a_{i 2}, \ldots, a_{i N_{i}}\right)^{T}, B_{i}=\left(b_{i 1}, b_{i 2}, \ldots b_{i N_{i}}\right)^{T}-\text { matrix-columns. }
\end{gathered}
$$

The elements of the antenna scattering matrix uniquely determine its characteristics as a component of the high-frequency path. However, the elements of the scattering matrix in the multiwave mode at non-fundamental frequencies are currently difficult to determine both theoretically and experimentally, so in practice, the so-called operating parameters are usually used to characterize the antenna. Of all the variety of their greatest interest is the transfer coefficient (power) of $i$-th shoulder in $j$-e.

\section{Control of characteristics of radio engineering systems in multi-wave mode}

In assessing the attenuation introduced by a multiwave antenna at non-operating frequencies, in the vast majority of cases, the modal composition of the signal coming to the input from the previous elements of the path of the radio system, the elements of the scattering matrix and, consequently, the modal compositions of the eigenvectors of the matrix are unknown $S_{j i}^{0} S_{j i}[7,8,9]$. When measuring the transmission coefficient, it is possible to fix the modal composition of the input signal, using, for example, exciters of wave types, but in this case we do not know the mutual orientation of the vector characterizing the modal composition of the input signal and the eigenvectors of the matrix $S_{j i}^{0} S_{j i}$. In both cases, the realized specific value of the transmission coefficient can be considered as a random realization of a possible set of values determined by the ratio.

The law of distribution of the transmission coefficient of the waveguide node on the set of possible generic compositions of the input signal can be obtained using the transmission coefficient connection $\beta_{k}$ :

$$
F\left(l_{i}\right)=\int_{\sum \lambda_{k}} \ldots \int_{\beta_{k}<l_{i}} f\left(\beta_{1}, \ldots, \beta_{N_{i}}\right) d \beta_{1} \ldots d \beta_{N_{i}},
$$

where $F\left(l_{i}\right)$ - the distribution of the transmission coefficient; $f\left(\beta_{1}, \ldots, \beta_{N_{i}}\right)$ - the joint probability density $\beta_{k}$. Integration into (6) should be conducted by area of definition $\beta_{k}(0 \ldots 1)$ subject to the limitations (2).

To estimate the joint probability density of quantities $\beta_{k}$ it is natural to proceed from the assumption of complete uncertainty of vector orientation $A_{i}$, characterizing the modal composition of the input signal relative to the eigenvectors of the matrix $S_{j i}^{0} S_{j i}$. It is enough to require execution for $\beta_{k}$ only one condition - the conditions of normalization (6). Then the joint probability density of the quantities $\beta_{k}$ can be represented as:

$$
f\left(\beta_{1}, \ldots, \beta_{N_{1}}\right)=\left(N_{i}-1\right) ! \theta\left(1-\sum_{1}^{N_{i}} \beta_{k}\right),
$$

where $\theta(x)$ - Delta function.

Substituting (7) in (6) and carrying out integration, we obtain: 


$$
F\left(l_{i}\right)=1+\sum_{k=1}^{N_{i}} \varphi\left(\frac{l_{i}}{\lambda_{k}}\right) \psi\left(\lambda_{k}\right)\left(1-\frac{l_{i}}{\lambda_{k}}\right)^{N_{i}-1}
$$

where

$$
\begin{gathered}
\varphi\left(\frac{l_{i}}{\lambda_{k}}\right)=\left\{\begin{array}{l}
0, l_{i} \geq \lambda_{k}, \\
1, l_{i}<\lambda_{k}
\end{array}\right. \\
\psi\left(\lambda_{k}\right)=\lambda_{k}^{N_{i}-1} \prod_{l \neq k}\left(\lambda_{i}-\lambda_{k}\right)
\end{gathered}
$$

Using asymptotic expressions for the psi-function and its first derivative, it is easy to obtain approximate expressions for the expectation, variance, and standard deviation of the value $L_{i}$ in decibels:

$$
\begin{gathered}
M\left(L_{i}\right)=-10 \lg M\left(l_{i}\right)+2,5-2,7 / N_{i}, \\
D\left(L_{i}\right)=31-18,9 / N_{i}-18,9 / 2 N_{i}^{2}, \\
\sigma\left(L_{i}\right)=5,57-2,2 / N_{i} .
\end{gathered}
$$

Values of values determined by these formulas for $N_{i}>2$, differ from the values obtained by the exact formulas (11) and (10) by no more than $0.1 \mathrm{~dB}$. At $N_{i} \rightarrow \infty$

$$
\begin{gathered}
M\left(L_{i}\right)+10 \lg M\left(l_{i}\right) \leq 2,5 \partial D, \\
\sigma\left(L_{i}\right) \leq 5,57 \partial Б,
\end{gathered}
$$

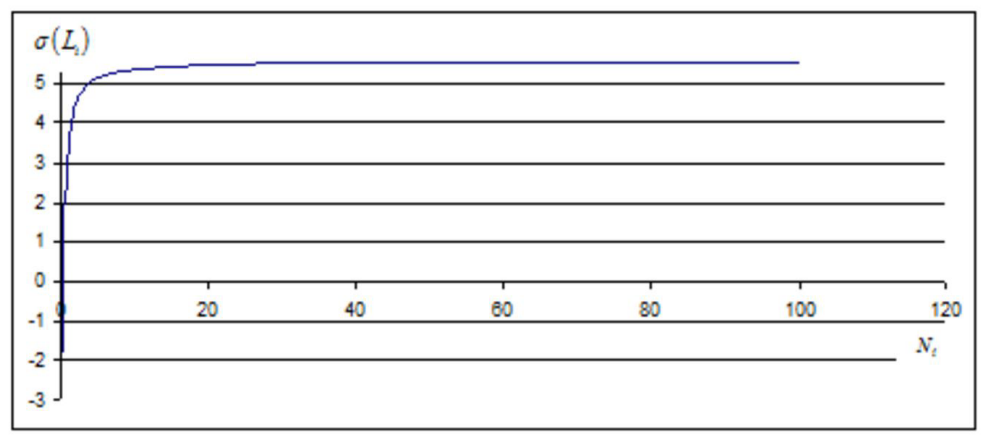

Fig. 1. Dependence of the mean square deviation on the number of wave types.

Thus, for mutual radio engineering systems in multiwave mode, the principle of reversibility can be formulated as follows:

1. The maximum value of the transfer coefficient from one shoulder is equal to the maximum value of the transfer coefficient from the other shoulder.

2. The mathematical expectation of the transfer coefficient from one arm is equal to the mathematical expectation of the transfer coefficient from the second arm only if the numbers of wave types that can propagate in one arm and the other are equal. In case of violation of this condition, the mutual waveguide nodes will have (on average) valve properties.

By asking the probability $P$, receive: 


$$
1 / V_{p}=M[1 / V(A)] N\left[1-(1-P)^{1 /(N-1)}\right]=M[1 / V(A)] \Delta P,
$$

where

$$
\left.\Delta P=N \mid 1-(1-P)^{1 /(N-1)}\right\rfloor .
$$

For fig. 2, the values of the correction factor $\Delta P$ little change in the number of types of waves that can propagate in the antenna of the radio system, so for all cases, you can use the limit values of the correction factor (when $N \rightarrow \infty$ ), equal 0,$11 ; 0,052$; and 0,020 accordingly, for the probability 0,$9 ; 0,95$ and 0,98 .

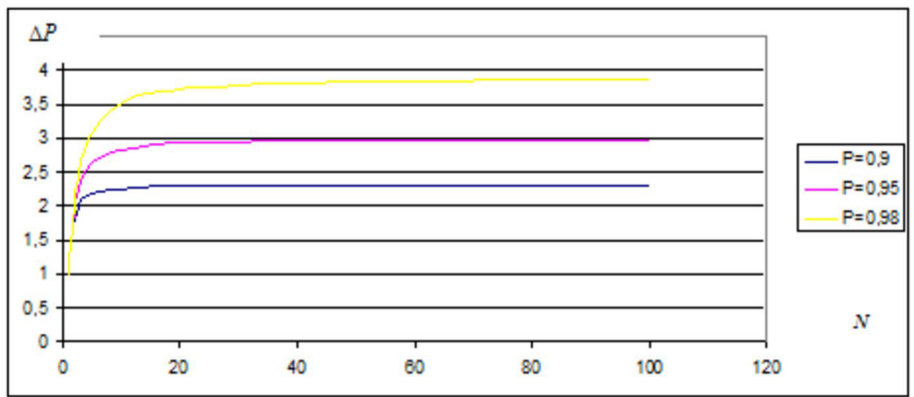

Fig. 2. Dependence of the correction factor on the number of wave types in the antenna system.

In the derivation of these relations, in addition to assumptions about the linearity of the characteristics of radio systems, used, in essence, another assumption about the absence of the modal current structure on the elements. In the UHF and microwave ranges, this assumption is carried out quite strictly, so the obtained ratios are valid for a wide class of radio systems [10].

\section{Conclusion}

The study illustrates the fact that even for simple input circuits of radio systems, the measurement of such a parameter as the level of susceptibility to test signals when the antenna is excited by a lower wave type can lead to an overestimation of the measurement results. The real input circuits of the radio system (especially when measured by the feeder) have different inhomogeneities: matching transformers, microwave filters, curved waveguide sections, switches, etc. All this makes it difficult a priori to determine the composition of the test signal at the input of the radio system, at which the lowest level of susceptibility is realized. Measurement of the level of susceptibility of the radio system at any fixed modal composition of the input test signal should in this case be considered as an implementation of the number of possible values. For a fairly wide class of input circuits of radio receivers all the set of values of the levels of susceptibility of the radio system in the multi-wave mode of its input circuits in statistical terms can be described quite fully by two parameters.

Therefore, the current distribution in the radio system does not depend on the modal composition of the test signal (in measurements) or interference signal (in operation) at the input of the radio system. Changing the modal composition of the input signal can only affect the amplitude and phase of the current in the antenna. The study summarizes the material needed to analyze the characteristics of radio systems 
obtained by the author. As a result of the analysis, calculations and proposals are made. In various ways to ensure the electromagnetic compatibility of electronic means the conclusion about the absence of unacceptable interference signal can be made by analyzing the data.

\section{References}

1. V. N. Pichugin, V. S. Gingerbread, Vestnik Kazanskogo state technical University , 31 (2009)

2. V. N. Pichugin, I. G. Bugrova, Scientific and information journal of the research Institute of education and science "Education. Science. Scientific personnel", 1, 134 (2013)

3. V. N. Pichugin, V. S. Gingerbread, Y. E. Sedelnikov, Antenna array of slotted radiators in the multiwave mode of admission: monograph (Izd-vo Chuvash. UN-TA, Cheboksary, 2013)

4. V. N. Pichugin, Big change: topical issues, achievements and innovations of social, humanitarian and economic development. Collection of scientific articles of the Regional scientific-practical conference, 55 (2017)

5. V. N. Pichugin, TECHNOLOGIES. Proceedings of the IX International scientific conference, 4 (2017)

6. V. N. Pichugin, R. V. Fedorov, M. P. Nemkova, M. A. Veryaskina, Ecology, Environment and Conservation, 21, AS1 (2015)

7. V. N. Pichugin, XXIII Tupolev readings (school of young scientists) international youth scientific conference: Proceedings of the conference. Tatarstan, 353 (2017)

8. V. N. Pichugin, Collection of scientific articles of the Regional scientific-practical conference with international participation, dedicated to the 50th anniversary of the Chuvash state University, Cheboksary, 118 (2017)

9. V. N. Pichugin, Current trends in the management of industrial innovation organizations Collection of scientific articles of the Regional scientific-practical conference with international participation dedicated to the 50th anniversary of the Chuvash state University, Cheboksaryry, 110 (2017)

10. E. N. Kadyshev, V. N. Pichugin, Problems and prospects of development of energy, electrical engineering and energy efficiency materials of the I International scientific and technical conference, Cheboksary, 171 (2017) 